\title{
SOME COMMENTS ON THE APPLICABILITY OF GAS PERMEATION METHODS TO CHARACTERIZE POROUS MEMBRANES BASED ON IMPROVED EXPERIMENTAL ACCURACY AND DATA HANDLING
}

\author{
F.W. ALTENA, H.A.M. KNOEF, H. HESKAMP, D. BARGEMAN and C.A. SMOLDERS \\ Department of Chemical Technology, Twente University of Technology, P.O. Box 217, \\ 7500 AE Enschede (The Netherlands)
}

(Received September 19, 1981; accepted in revised form July 19, 1982)

\section{Summary}

The measurement of the gas permeability coefficient as a function of the mean pressure across a membrane can be used to determine a mean pore radius of the membrane. This method has been applied by several authors to characterize microporous and asymmetric ultrafiltration or hyperfiltration membranes. This paper shows how the data acquisition and handling are improved. Experiments are performed on microporous Millipore membranes with a nominal pore radius of $50 \mathrm{~nm}$ and on ultrafiltration membranes of poly(2,6-dimethyl-1,4-phenyleneoxide) with an expectedly sharp pore-size distribution around $2 \mathrm{~nm}$. For the Millipore membrane an unexpected dependence of the calculated pore radius on the type of gas used in the experiment has been found. Measurements on the ultrafiltration membranes indicate that the viscous flow contribution to the permeability coefficient cannot be determined with sufficient accuracy. It is concluded that application of the gas permeation method has some limitations which were not previously recognized.

\section{Introduction}

The measurement of the flow rate of a gas through a porous medium can provide a means of determining a mean pore radius of the porous material. In recent years several authors [1-3] have used the method suggested by Yasuda and Tsai [4] to characterize ultrafiltration or hyperfiltration (reverse osmosis) membranes. The flow rate of the gas can be converted to a gas permeability coefficient, which is a linear function of the mean pressure across the membrane. A mean pore radius is calculated from the coefficients of this functional dependence, using theoretical equations originally given by Carman [5]. Although the value for the pore radius derived in this way may not have a precisely defined physical meaning, it is considered a useful tool when comparing membranes prepared in slightly different preparation procedures.

The purpose of this study was to apply the method to ultrafiltration membranes of poly (2,6-dimethyl-1,4-phenyleneoxide) ( $\mathrm{PPO}^{\otimes}$ ) having a sharp pore-size distribution around $2 \mathrm{~nm}$ [6]. Determination of this distribution 
involved rather tedious gas adsorption experiments. The gas permeation method of Yasuda appeared to be an elegant way of characterizing the pore structure of the prepared membranes. One of the main characteristics of this method is that the derived value of the equivalent pore radius does not depend on the type of gas used in the experiment. However, results of experiments, intended to reproduce some of Yasuda's results and described in this paper, do not confirm this.

It is assumed that in the microporous pore size region (say pore radius $>30 \mathrm{~nm}$ ), with a fixed pore structure, both viscous and slip flow contribute to the gas flow through the membrane. Carman's equations are used in this case.

At much smaller pore radii (in nm range) other mechanisms such as solution-diffusion may have important contributions. We will confine ourselves to the treatment of Yasuda and other authors [1-4] and ignore this mechanism. Thus for ultrafiltration, where the pore concept is expected to have a physical meaning, and for reverse osmosis membranes, where this is less likely, it is assumed that viscous flow is operative, and again the equations of Carman are supposed to describe the gas flow through the membrane.

Some peculiar results have been presented by authors who have applied $Y$ asuda's method [2-4] . For polysulfone ultrafiltration membranes no pore radius below $25 \mathrm{~nm}$ is reported [2,4]. This value for standard ultrafiltration membranes is, in our opinion, not very realistic. Kakuta et al. [3] have given a pore diameter of 3-4 $\mathrm{nm}$ for a cellulose acetate reverse osmosis membrane. From their experimental plot of the permeability coefficient versus the mean pressure one can calculate, however, a pore radius of $8 \mathrm{~nm}$ for nitrogen. For an effective cellulose acetate hyperfiltration membrane this seems to be rather high. The gas permeation method may yield a rather high average pore radius for the membranes considered. A second problem is indicated by Kakuta's results. The data points for helium as the gas used in the experiment are scattered, and a much smaller pore radius value would also fit the experimental data. Clearly there appears to be a problem in obtaining sufficiently accurate experimental data.

In this paper the basic equations are first reviewed. An improved way to handle the experimental data is presented. Next the problem of obtaining sufficient accuracy in the measurement of the pressure decay across the membrane is investigated. Accurate pressure measurements are needed to be able to calculate a reliable value for the average pore radius. For the case of microporous membranes the experimental set-up and the data acquisition meet the requirements.

\section{Theoretical considerations}

For the flow of gas through a porous structure Darcy's law takes the 
following form $[5 \mathrm{a}, 7 \mathrm{a}]$ :

$v_{2}=\frac{B_{0} \bar{p} \Delta p}{\eta p_{2} l}$

where $v_{2}$ is the flow velocity of the gas at (outlet) pressure $p_{2} ; \Delta p=p_{1}-p_{2}$ is the pressure difference across the membrane; $\bar{p}=1 / 2\left(p_{1}+p_{2}\right)$ is the mean pressure; $B_{0}$ is the specific permeability coefficient; $\eta$ is the viscosity and $l$ is the thickness of the membrane. For the gas flux through the membrane we can write, if we combine constant factors:

$J=K \frac{\Delta p}{l}$

where $K$ is the permeability coefficient. If the mean pore diameter is comparable in size to the mean free path $(0.01-0.1 \mu \mathrm{m}$ at room temperature and atmospheric pressure) $K$ contains another (non-viscous) term. This effect is due to the so-called slip effect, and can be included in:

$K=K_{0}+\frac{B_{0}}{\eta} \bar{p}$

$K_{0}$ and $B_{0}$ are characteristic of the porous medium and the gas.

The gas flux is measured by the pressure decay in a gas ballast chamber of fixed volume $V_{0}$ connected to the membrane test cell.

Following Yasuda [4] :

$J=\frac{\mathrm{d}(p V)}{\mathrm{d} t}=-V_{0} \frac{\mathrm{d}(\Delta p)}{\mathrm{d} t}$

Combining eqns. (2), (3) and (4) gives, after deleting constant factors:

$-\frac{\mathrm{d}(\Delta p)}{\mathrm{d} t}=\left(K_{0}+\frac{B_{0}}{\eta} \bar{p}\right) \Delta p$

with $\vec{p}=1 / 2\left(p_{1}+p_{2}\right)=1 / 2\left(\Delta p+2 p_{2}\right)$, where in our case $p_{2}$ is the atmosphere pressure. Equation (5) becomes:

$-\frac{\mathrm{d}(\Delta p)}{\mathrm{d} t}=\left(K_{0}+\frac{B_{0}}{\eta} p_{2}\right) \Delta p+\frac{B_{0}}{2 \eta}(\Delta p)^{2}$

The flux equation has been rewritten as a differential equation for the pressure difference across the membrane. For simplicity we write $p=\Delta p, a=$ $K_{0}+\left(B_{0} / \eta\right) p_{2}$ and $b=B_{0} / 2 \eta$. Equation (6) becomes:

$-\frac{\mathrm{d} p}{\mathrm{~d} t}=a p+b p^{2}$

In the foregoing, constant terms have been omitted because only the ratio of $a$ and $b$ defines the pore radius to be calculated (cf. eqn. (12)). 
Shimizu [1], Cabasso [2], Kakuta [3] and Yasuda [4] all follow Carman [5b] by putting:

$\begin{aligned} B_{0} & =\frac{\epsilon m^{2}}{k_{0} q^{2}} \\ K_{0} & =\frac{4 \epsilon \delta m}{3 k_{1} q^{2}} \bar{v}\end{aligned}$

where $m$ is the equivalent pore radius, $\epsilon$ is the porosity, $q^{2}$ the tortuosity factor and $k_{0}$ and $\delta / k_{1}$ are assumed to be constants (Carman [5b] suggested the values of $k_{0}=2.5$ and $\left.\delta / k_{1}=0.8\right) . \bar{v}$ is the average molecular velocity of the gas with molecular weight $M$ :

$\bar{v}=\left(\frac{8 R T}{\pi M}\right)^{1 / 2}$

Combining eqns. (8), (9) and (10) gives:

$m=\frac{16}{3}\left(\frac{B_{0}}{K_{0}}\right)\left(\frac{2 R T}{\pi M}\right)^{1 / 2}$

or when using $a$ and $b$ defined by eqn. (7):

$m=\frac{16}{3}\left(\frac{2 \eta b}{a-2 b p_{2}}\right)\left(\frac{2 R T}{\pi M}\right)^{1 / 2}$

This is the desired pore radius to be calculated from the experiments.

\section{Improved data handling}

In the experiments the pressure decay $p=p(t)$ is measured. Yasuda and Tsai [4] determine $\mathrm{d} p / \mathrm{d} t$ from this decay recorded on a strip chart recorder. $K_{0}$ and $B_{0}$ can be determined from eqn. (5) using the slope and intercept and from eqn. (11) $m$ can then be calculated.

We have taken a different approach by rewriting eqn. (7) and integrating from chosen points of $p=p(t)$, viz. $p_{0}$ at $t=t_{0}$ and $p_{e}$ at $t=t_{e}$ :

$\int_{p_{0}}^{p_{e}} \frac{\mathrm{d} p}{a p+b p^{2}}=-\int_{t_{0}}^{t_{e}} \mathrm{~d} t$

It follows:

$\frac{1}{a}\left[\ln \left(\frac{a+b p_{e}}{p_{e}}\right)-\ln \left(\frac{a+b p_{0}}{p_{0}}\right)\right]=t_{e}-t_{0}$

In principle we need only two points $\left(t_{e}, p_{e}\right)$ at fixed $\left(t_{0}, p_{0}\right)$ of the pressur decay to solve $a$ and $b$ from non-linear equations of the type (14). We prefer 
this method of data analysis to the one used by Yasuda because the pressure itself can be determined more accurately than $\mathrm{d} p / \mathrm{d} t$.

In practice it is better to take more points $\left(t_{e}(i), p_{e}(i), i=2, \ldots, n\right)$ and to evaluate at least $10(n>10)$ equations (14) to calculate $a$ and $b$. This is done by a numerical procedure using a non-linear least squares algorithm which solves $n$ equations simultaneously. The choice of the points on the pressure decay curve could be a source of inaccuracy. We have tried two procedures:

- A fixed $\left(t_{0}, p_{0}\right)$ is chosen, about $(0 \mathrm{~s}, 3.5 \mathrm{bar})$, and $n$ points $\left(t_{e}, p_{e}\right)$ on the curve are equally spaced in time; $n$ equations (14) are solved for each $\left(t_{e}, p_{e}\right),\left(t_{0}, p_{0}\right)$ pair.

- In the second procedure the point $\left(t_{0}, p_{0}\right)$ is varied; $n$ points are chosen on the pressure decay curve, and eqn. (14) is solved with pairs $\left(t_{1}, p_{1}\right),\left(t_{n / 2+1}\right.$, $\left.p_{n / 2+1}\right)$ and $\left(t_{2,} p_{2}\right),\left(t_{n / 2+2}, p_{n / 2+2}\right)$ etc. $(n$ even $>4)$. These two procedures give the same results.

When $a$ and $b$ have been calculated the experimental $p=p(t)$ curve is compared with the calculated one. From eqn. (14) it follows:

$$
p=\frac{a}{\exp \left\{-a\left(t_{0}-t\right)+C\right\}-b}
$$

with

$C=\ln \left(\frac{a}{p_{0}}+b\right)$

\section{Required accuracy of pressure measurements}

In the experiments the pressure difference across the membrane $p$ decreases from $p_{0}$ (about 3.5 bar) to zero. As will be seen below, $b<<a$. Thus, when $p$ is small, $\mathrm{d} p / \mathrm{d} t$ is mainly determined by $a$ and a high precision of $b$ cannot be expected (see eqn. (7)).

In the case of Millipore VM type membranes the following approximate values of $a$ and $b$ have been found (see Results): Helium: $a=0.2, b=0.002$; Nitrogen: $a=0.1, b=0.01$. From eqn. (7) we see that a relative error $\Delta p / p$ of 0.01 gives a relative error in $b$ and consequently in $m$ (cf. eqn. (12)) of $\Delta b / b$ of 0.1 for nitrogen and 0.5 for helium (at $p=1$ bar; $\mathrm{d} p / \mathrm{d} t$ held constant). A precise determination of $b$ (or $m$ ) can only be expected when $\Delta p / p=0.001$. In this case the respective relative errors are $0.01\left(\mathrm{~N}_{2}\right)$ and $0.05(\mathrm{He})$. We conclude that a very precise measurement of $p$, the pressure difference across the membrane, is required. Yasuda and Tsai [4] derive values for the pressure gradient from a strip chart recorder. The reading error thus introduced is of the order of 0.01 . No precise determination can be expected. An absolute precision of about 0.003 bar is required to obtain $m$ with sufficient accuracy. 


\section{Experimental}

The experimental set-up is shown in Fig. 1. The pressure decay in the reservoir $\left(0.01 \mathrm{~m}^{3}\right)$ is measured by the pressure transducer (Viatran 304 , 0-150 PSIA, $3000 \mu \mathrm{V}$ FSO). The ballast chamber is pressurized to a pressure of about 5 bar. The valve between gas cylinder and ballast chamber is closed, the ball-valve between the ballast chamber and the cell with the membrane is opened and the pressure decay is recorded. In order to avoid an initial transient stage of pressure decay, the chamber is pressurized to a pressure $50 \%$ higher than the pressure ( $3.5 \mathrm{bar})$ needed in the measurement [4]. The gas is allowed to flow through the membrane before the pressure reading is taken. We have used a Millipore filter holder XX 4502500.

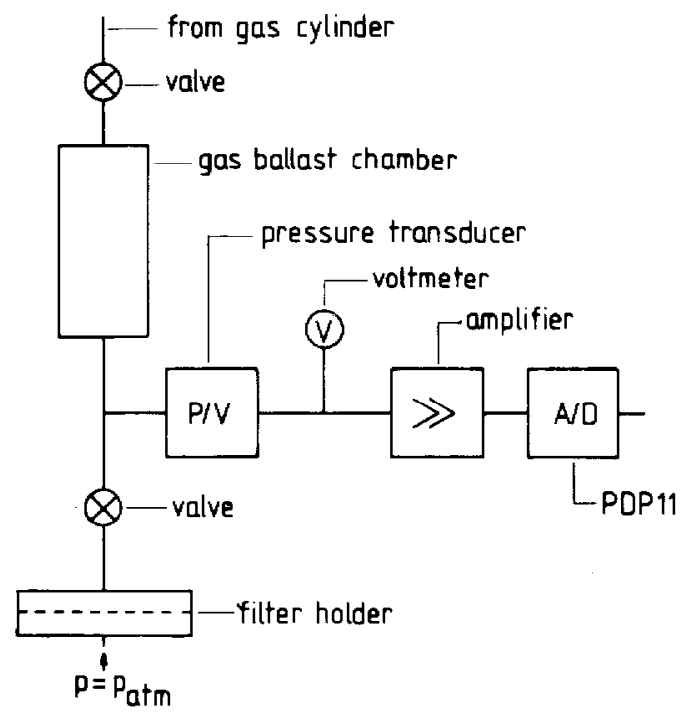

Fig. 1. Fxperimental set-up for gas permeation experiment.

The pressure transducer is coupled on line to the A/D converter of a PDP 11 minicomputer. Amplification of the signal of the pressure transducer was necessary (Tektronix AM 502, 200 $\times$ ). The data of the minicomputer were handled off-line to calculate the equivalent pore radius. Calibration of the $A / D$ readings versus the signal of the pressure transducer (digital voltmeter, HP $3465 \mathrm{~A}$ ) was performed. The uncertainty in the static pressure measurement appeared to be $\pm 0.003 \mathrm{bar}$. The initial pressure decay is about 0.01 $\mathrm{bar} / \mathrm{s}$. The A/D converter averages 16 samples of the pressurc signal (total time $8 \mathrm{~ms}$ ), and gives no extra contribution to the uncertainty.

The absolute pressure reading of the pressure transducer is calibrated with an Industrial Air Dead Weight Tester of Barnet, type 3320 (10-1000 psi), which has a sensitivity of $3 \times 10^{-3}$ bar. The deviation of linearity of the pressure transducer is about this value and well within the specifications of the manufacturer. 
The experiments were performed in a thermostated room at $T=20^{\circ} \mathrm{C}$. The atmospheric pressure is read from a Lambrecht mercury column manometer, reading error $\pm 2 \times 10^{-4}$ bar.

\section{Results}

Experiments were performed on a Millipore type VM membrane and on PPO ultrafiltration membranes. The Millipore membrane has a nominal pore radius of $50 \mathrm{~nm}$, given by the manufacturer. The PPO ultrafiltration membranes were made as described by Broens et al. [6] .

Using the procedure described in the section on data handling we calculated values for $a$ and $b$ for the Millipore membrane from the pressure decay curve from 20 or 40 equations of the type (14). We have used three gases: helium, nitrogen and carbon dioxide. The pressure readings were taken at intervals of $0.5 \mathrm{~min}$ over $20 \mathrm{~min}$. The calculated values for $a, b$ and the resulting pore radius $m$ are given in Table 1 .

The given inaccuracy has been estimated by choosing $a$ and $b$ values such that $F<5 \times 10^{-4}$, where

$F=\sum_{n=1}^{n=40}\left(p_{\text {calc }}\left(t_{n}\right)-p_{\exp }\left(t_{n}\right)\right)^{2}$.

We have used eqns. (15) and (16) to determine $p_{\text {calc }}\left(t_{n}\right)$. This value of $F$ means that the difference $\left|p_{\text {calc }}\left(t_{n}\right)-p_{\exp }\left(t_{n}\right)\right|$ for each point is about $3 \times 10^{-3}$ bar, the uncertainty in the pressure measurement.

Application of the above-described procedure to the PPO membranes did not yield reliable results. It appears to be very difficult to determine the constant $b$, a measure for the viscous flow contribution to the gas flow through the membrane. In some cases even physically unrealistic negative values of the constant $b$ (see eqn. (12)) were found.

TABLE 1

Pore radius of a Millipore VM membrane

\begin{tabular}{llll} 
& $a$ & $b$ & $m(\mathrm{~nm})$ \\
\hline Helium & $0.237 \pm 0.003$ & $0.0029 \pm 0.001$ & $15 \pm 5$ \\
Nitrogen & $0.108 \pm 0.002$ & $0.0084 \pm 0.0007$ & $40 \pm 4$ \\
Carbon dioxide & $0.094 \pm 0.005$ & $0.010 \pm 0.001$ & $38 \pm 4$ \\
\hline
\end{tabular}

\section{Discussion}

\section{Microporous membranes}

When we started our experiments we considered Yasuda's method a useful tool to characterize porous membranes. Determination of $\mathrm{d} p / \mathrm{d} t$ from the pressure decay curve seemed to us rather inaccurate. This problem could be 
circumvented by solving a set of non-linear equations in which the pressure itself appears instead of the derivative. After solving this problem it became evident that very accurate pressure measurements were needed to obtain sufficient accuracy in the equivalent pore radius. Above we have given the results of sufficiently accurate measurements.

Contrary to Yasuda's results we have found that the pore radius for a Millipore VM membrane depends on the type of gas used. Approximately the same value results when nitrogen or carbon dioxide are used, which is in agreement with Yasuda. For helium we find a much smaller value (He: $15 \mathrm{~nm}$; $\mathrm{N}_{2}$ and $\mathrm{CO}_{2}: 40 \mathrm{~nm}$ ). This difference cannot be due to lack of accuracy of the measurements. To obtain a pore radius for helium of about $40 \mathrm{~nm}$ one would have to allow for a systematic error in the pressure measurement of as large as 0.03 bar. The expected accuracy is about 10 times better.

The dependence of the pore size on the type of gas used in the experiment complicates, in our opinion, the applicability of Yasuda's method. An explanation of this effect may be as follows.

In using the equations of Carman we assume that both terms of the permeability coefficient, the viscous flow and the slip contribution, are present. Considering the mean free path of the three gases used (He:270; $\mathrm{N}_{2}: 90$ and

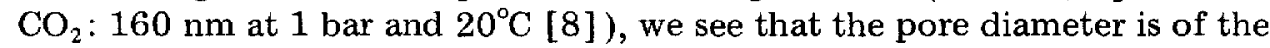
order of the mean free path. If there should be a large number of pores of a much smaller diameter the situation would be different. These pores contribute much less to the viscous flow through the membrane but may contribute a relatively higher amount to the non-viscous flow. As we know, the permeability coefficient should be a linear function of the mean pressure. For many porous media $K$ passes through a minimum at small values of $\bar{p}$, when the region of Knudsen flow is reached [7b]. This region is reached faster for helium because of the larger mean free path. The same reasoning applies to $K$ as a function of the pore radius. Therefore, if a large number of small pores is present, then there is a large contribution of these pores to the non-viscous term of the permeability coefficient, and an apparently smaller pore radius will be found. In this way a complex averaging over the pore size distribution occurs in the gas permeation experiment.

It should be noted that Shimizu et al. [1] do find consistent results for all gases considered ( $\mathrm{Ar}, \mathrm{O}_{2}, \mathrm{He}, \mathrm{H}_{2}$ and $\mathrm{CO}_{2}$ ) in the range of $40-80 \mathrm{~nm}$ for porous propylene membranes. More experiments are needed to investigate whether the found dependency on the type of gas used stems from a certain special pore size distribution. Maybe the reported observation can be turned to good use in the characterization of a membrane.

\section{Ultrafiltration and hyperfiltration membranes}

Extending our studies to the pore size region where top layers of ultrafiltration or hyperfiltration have their expected pore radius adds another problem, i.e. to determine the convective term of the permeability coefficient with sufficient accuracy. Some simple calculations using eqn. (15) 
show that we cannot discriminate between pore radii of 2 and $4 \mathrm{~nm}$ : as far as the accuracy of the pressure measurement is concerned the convective contribution could equally be absent. This is in agreement with the fact that no reliable value for the pore radius (of PPO membranes) could be found.

Let us assume that the experimental accuracy is adequate to allow for a reliable determination of an average pore radius. If small pores do not contribute to the convective flow, an averaging takes place over the relatively large pores. Reported pore radii of $25 \mathrm{~nm}$ for polysulfone and $8 \mathrm{~nm}$ for cellulose acetate membranes point in that direction. This contention has to be supported by more experiments on well-defined membranes with a sharp size distribution, preferably using several techniques.

A similar discrepancy, i.e. the dependence of the pore radius on the type of gas used, as has been observed with the Millipore membranes, can be seen from the plots of the permeability coefficient against the mean pressure of Kakuta et al. [3] . A smaller value for helium than for nitrogen for the pore radius may also fit the inaccurate data.

As has been mentioned before, a model description based only on pore flow mechanisms may fail to describe the gas transport in the "pores" at the lower (reverse osmosis) end of the pore size spectrum. Adoption of a flow mechanism not based on flow through pores, such as solution-diffusion, is required. This is beyond the scope of the present paper.

\section{Conclusions}

The data acquisition and handling of the pressure decay curve in a gas permeation experiment are considerably improved.

The results of the experiments point out some limitations of the gas permeation method:

- The value of the mean pore radius may depend on the type of gas used in the experiments. This is established for one type of Millipore membrane.

- The accuracy of the determination of the convective contribution to the gas flow does not allow a reliable determination of the average pore radius of an (PPO) ultrafiltration membrane with a narrow pore size distribution.

\section{Acknowledgement}

The authors are grateful to Dr. M. Bos for his valuable assistance with the on-line pressure measurements.

\section{Notation}

$a$ defined by eqns. (6) and (7)

$\mathrm{m}^{2} \mathrm{~s}^{-1}$

$b$ defined by eqns. (6) and (7)

$\mathrm{m}^{2} \mathrm{~s}^{-1}$

$B_{0}$ specific permeability coefficient

$J$ gasflux through membrane

$\mathrm{m}^{2}$

$\mathrm{kg} \mathrm{m}^{-2} \mathrm{~s}^{-1}$ 
$K$ permeability coefficient, defined by eqn. (3)

$\mathrm{m}^{2} \mathrm{~s}^{-1}$

$K_{0}$ Knudsen permeability coefficient

$\mathrm{m}^{2} \mathrm{~s}^{-1}$

$k_{0}$ constant, eqn. (8)

$k_{1}$ constant, eqn. (9)

$l$ thickness of membrane $\mathrm{m}$

$m$ mean pore radius $\mathrm{m}$

$M$ molecular weight

$\Delta p$ pressure difference across membrane, $\Delta p=p_{1}-p_{2}$

$\mathrm{kg} \mathrm{mol}{ }^{-1}$

$p$ pressure difference across membrane, $p=\Delta p$

$\vec{p}$ mean pressure, $\bar{p}=1 / 2\left(p_{1}+p_{2}\right)$

bar

bar

$p_{1}$ inlet pressure

bar

$p_{2}$ outlet pressure

$p_{0}$ points on pressure decay curve $\left(t_{0}, p_{0}\right)$

bar

bar

$p_{e}$ points on pressure decay curve $\left(t_{e}, p_{e}\right)$

bar

$q$ tortuosity factor

$R$ universal gas constant

$T$ absolute temperature

$t_{0}$ point on pressure decay curve $\left(t_{0}, p_{0}\right)$

bar

$t_{e}$ point on pressure decay curve $\left(t_{e}, p_{e}\right) \quad \mathrm{s}$

$t$ time

$V$ volume gas ballast chamber

$8.314 \mathrm{~J} \mathrm{~mol}^{-1} \mathrm{~K}^{-1}$ $\mathrm{K}$

$\bar{v}$ average molecular velocity

$v_{2}$ flow velocity of gas at pressure $p_{2}$ 